\title{
Development of the Flight Tether for ProSEDS
}

\author{
Leslie Curtis ${ }^{1}$, Jason Vaughn ${ }^{1}$, Ken Welzyn ${ }^{1}$, Joe Carroll ${ }^{2}$ \\ ${ }^{1}$ Space Transportation Directorate, National Aeronautics and Space Administration, Marshall Space Flight Center, \\ AL 35812, ${ }^{2}$ Tether Applications, Inc., 1813 Gotham St. Chula Vista, CA 91913-2624 \\ 256-544-2486, leslie.curtis@msfc.nasa.gov,256-544-9347,jason.vaughn@msfc.nasa.gov,256-544-1731, \\ ken.welzyn@msfc.nasa.gov,619-421-2100,tether@home.com
}

\begin{abstract}
The Propulsive Small Expendable Deployer System (ProSEDS) space experiment will demonstrate the use of an electrodynamic tether propulsion system to generate thrust in space by decreasing the orbital altitude of a Delta II Expendable Launch Vehicle second stage. ProSEDS will use the flight-proven Small Expendable Deployer System to deploy a newly designed and developed tether which will provide tether generated drag thrust of $\sim 0.4 \mathrm{~N}$. The development and production of very long tethers with specific properties for performance and survivability will be required to enable future tether missions. The ProSEDS tether design and the development process may provide some lessons learned for these future missions. The ProSEDS system requirements drove the design of the tether to have three different sections of tether each serving a specialized purpose. The tether is a total of 15 kilometers long: 10 kilometers of a non-conductive Dyneema lead tether; $5 \mathrm{~km}$ of CCOR conductive coated wire; and 220 meters of insulated wire with a protective Kevlar overbraid. Production and joining of long tether lengths involved many development efforts. Extensive testing of tether materials including ground deployment of the full-length ProSEDS tether was conducted to validate the tether design and performance before flight.
\end{abstract}

\section{PROSEDS MISSION OVERVIEW}

ProSEDS is an electrodynamic tether propulsion system space experiment scheduled to fly in 2002 as a secondary payload on a Delta II Global Positioning System (GPS) replacement mission. After the primary payload is delivered, the ProSEDS mission begins. First, the Delta second stage is placed in a $360 \mathrm{~km}$ circular orbit with a 36 $+/-1$ degree inclination. An endmass weighing approximately $21 \mathrm{~kg}$ is then deployed upward (away from the earth) by spring ejection from the Delta at an initial rate of $3 \mathrm{~m} / \mathrm{s}$. The first $10 \mathrm{~km}$ of tether, which is connected to the endmass, is a nonconductive material which provides the gravity gradient force required to deploy the remaining conductive portion of the tether. The deployer control system applies appropriate braking to bring the total tethered system to a vertical stable orientation at the end of deployment. The end of the $5 \mathrm{~km}$ conductive tether remains attached to the Delta II. As the system moves through the earth's magnetic field, a motional-induced potential attracts electrons from the surrounding plasma. The electrons are collected by the conductively coated tether and flow to the Delta stage where a plasma contactor is used to emit them back into space to complete the circuit through the space plasma. A force is exerted on the current-carrying tether by the earth's magnetic field which causes the altitude of the Delta to decrease. The Delta stage, with ProSEDS attached, will continue it's orbital decay until it burns up upon reentry into the atmosphere. The ProSEDS experiment will demonstrate electrodynamic tether thrust capability by lowering the orbit of the stage by at least $5 \mathrm{~km}$ a day. Instrumentation on ProSEDS will measure the plasma density and other conditions to determine the current collection capability of the electrodynamic tether. The data obtained from the ProSEDS experiment will be used to predict the performance of future tether missions. 


\title{
TETHER DEPLOYER SYSTEM DESCRIPTION
}

The tether deployer system for ProSEDS is based on the flight proven Small Expendable Deployer System (SEDS) deployer, which has flown successfully three times. The deployer has an aluminum canister that houses the tether winding on an aluminum core. The canister also houses phototransistors and infrared light emitting diodes that act as counters to monitor the tether length and rate during deployment. The tether for the ProSEDS experiment is 15 $\mathrm{km}$ long with three distinct sections connected together: a non-conducting section, an uninsulated conducting section, and an insulated conducting section (Fig. 1). The 220 meter insulated section is secured to the tether core in the deployer assembly, which remains attached to the Delta II second stage during the entire mission. This section is insulated to prevent any electron reconnection to the tether from the plasma contactor. It is also is covered with a Kevlar overbraid for wear and handling protection. Both the insulated and uninsulated conductive sections combined comprise $5 \mathrm{~km}$ and consist of 7 continuous strands of 28 AWG aluminum wire twisted around a Kevlar core. The Kevlar core provides ample tensile strength for the tether and improved windability and deployability characteristics. For the uninsulated section, the aluminum wire strands are coated with a conductive atomic oxygen tolerant polymer to provide good surface conductivity (for electron collection) and improved surface optical properties to lower temperatures on orbit. Connected to the conductive section of tether is a $10 \mathrm{~km}$ long tether made from Dyneema fiber braided into a flat geometry to provide improved resistance to meteoroid impact. (This end of the tether is deployed first, as its low friction deployment properties are needed to allow enough tether to be deployed by the ejection momentum of the endmass until sufficient gravity gradient forces develop. Also, a conductive connection to the endmass is not required. This tether also provides a stabilizing tension force to the conductive portion of the tether, which will be subjected to horizontal forces much like the wind pushing on a sail.) The entire tether passes through the brake and ammeter, which are mounted above the canister, as it is deployed. The brake consists of a post and tether guide that can be rotated under the action of a stepper motor (commanded by the deployment control law) to control the tether tension and hence the deployment rate. The deployer is assembled at MSFC with other hardware (Fig 2). The deployer canister and brake subsystem were designed and fabricated by Tether Applications Inc. of Chula Vista, CA.

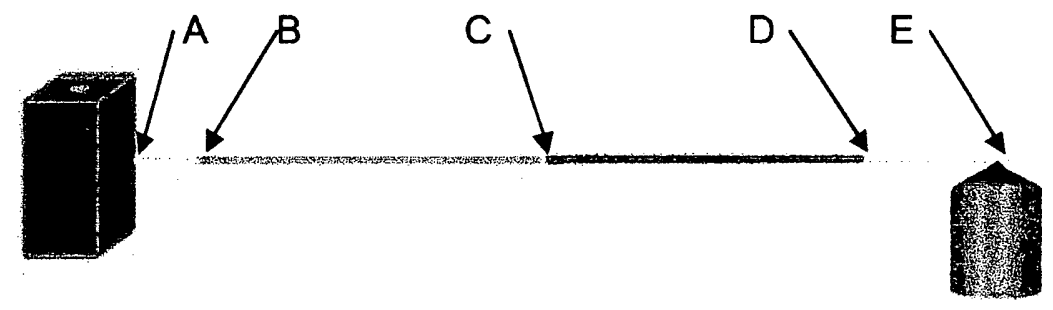

\author{
Non-Conductina Tether: $10 \mathrm{~km}$. Section A-C \\ Section A-B: $20 \mathrm{~m}$ Kevlar Leader \\ Section B-C: $10 \mathrm{~km}$ Dvneema Flat Braid \\ Conductina Tether: $5220 \mathrm{~m}$. Section C-E \\ Section C-D: 5 km CCOR Coated Aluminum Wire. Kevlar Core \\ Section D-E: 220 m Insulated Aluminum Wire. Kevlar Core. Overbraid
}

FIGURE 1. ProSEDS Tether Schematic.

Upon command from Delta the endmass is ejected from the second stage pulling the tether from the canister, initiating deployment. The brake is applied at various times during deployment to control the deployment rate of the tether. The brake control law is a modification of what was used on previous SEDS mission. The control law is finalized and tested numerous times during deployment testing of flight type tethers in a vacuum chamber at MSFC. The control law and brake settings are preprogrammed into the data subsystem electronics box before launch 
because there is no uplink command capability. All of the data on turns counts, tension, and deployment will be transferred to ground stations during the mission for post mission analysis.

\section{TETHER DESIGN}

The design of the tether for the ProSEDS experiment was based on analysis of the space environment for the experiment including the ionospheric plasma conditions, meteoroid and orbital debris, natural thermal environments, radiation and solar conditions, and atomic oxygen. System trades were performed on tether lengths and materials, and many tests were conducted of various material and tether samples to finalize the design. In addition to trades and analysis on the tether performance, the decision to use the SEDS flight proven hardware provided several constraints to the tether design. These constraints included volume limitations of the canister and mass limitations of the canister and support structure.

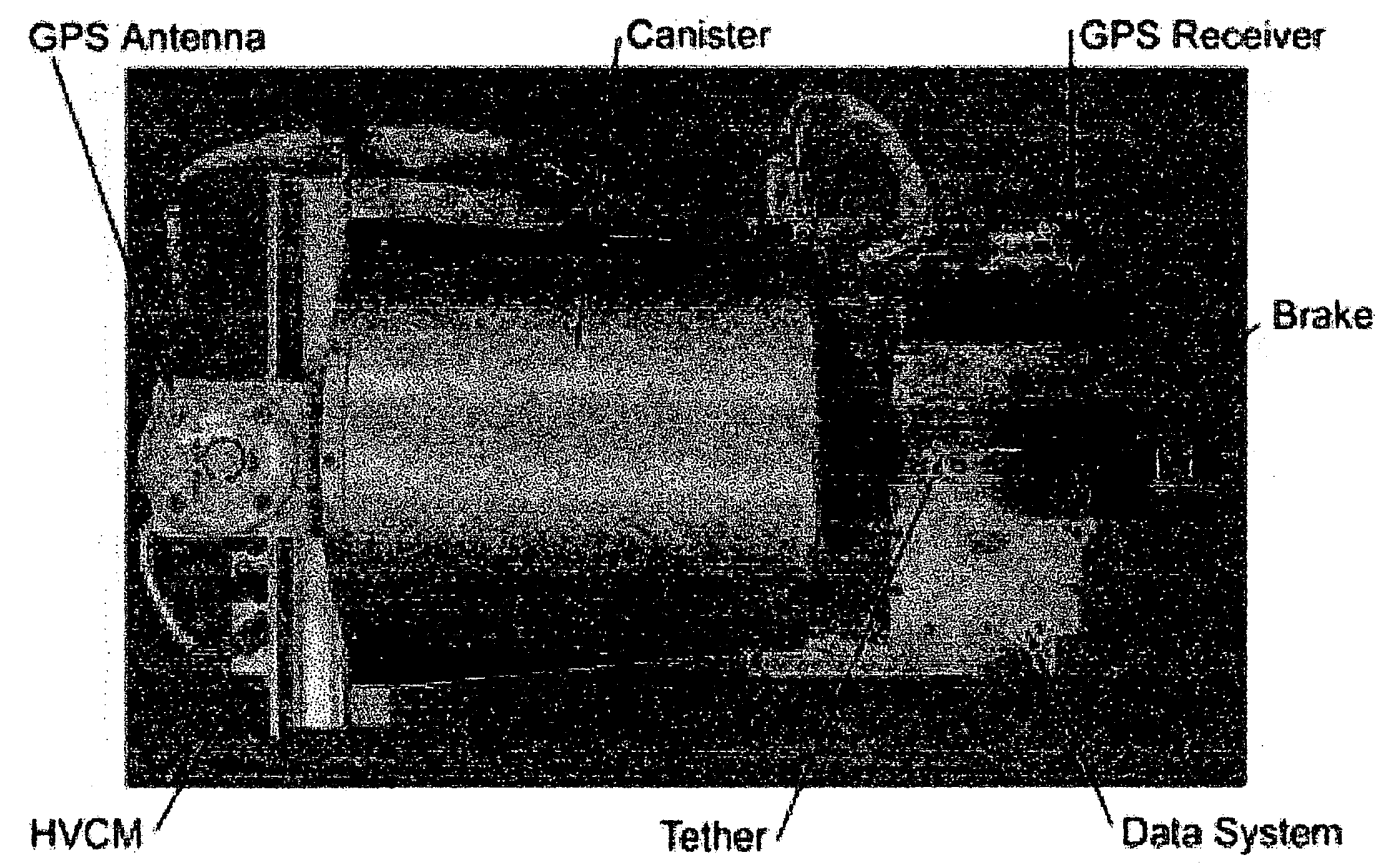

FIGURE 2. ProSEDS Deployer Hardware Assembly.

Since previous tether missions had successfully deployed non-conducting tethers of $20 \mathrm{~km}$ lengths from SEDS hardware, the decision to use a $10 \mathrm{~km}$ non-conducting tether for initial deployment and for tether stabilization was made. The one change that was made was to use a flat $(1.2 \mathrm{~mm} \times 0.16 \mathrm{~mm})$ braided tether instead of a cylindrical tether to improve the survivability of the non-conducting tether in case of impact with a micro-meteoroid particle.

An analysis of the ionospheric plasma conditions that would be present in space during the mission was conducted to determine the electromotive force (EMF) that could be generated by various lengths and sizes of metallic tether. The outer diameter of the conductive tether needed to be around $1.2 \mathrm{~mm}$ in order to provide the needed current collecting area while fitting the volume constraints of the SEDS canister. Lengths that were considered were 3, 4, and $5 \mathrm{~km}$. It was determined that a length of $5 \mathrm{~km}$ would be required to generate a tether current of $3 \mathrm{~A}$. Shortening the tether to save system mass would lower the current collection capability of the system and limit the demonstration of the electrodynamic tether generated forces. The maximum value of tether generated EMF for ProSEDS is predicted to be $1400 \mathrm{~V}$, which will provide an ample demonstration of the electrodynamic tether thrust capability of tethers. 
The $5 \mathrm{~km}$ conducting tether uses seven wires twisted around a high strength braided core. This is a proven design for production of long length cables and provides a flexible, strong (greater than $250 \mathrm{~N}$ tensile strength) tether for winding and deployment. Trades were conducted on the metallic material to be used for the conducting portion of the tether. The two materials that were studied in depth were copper and aluminum. Some of the considerations included: current collection capability, system mass, material deployability, manufacture and handling of the tether. Comparisons were made for the current collection capability of copper and aluminum wire. If a slightly larger diameter aluminum wire is used the current collected is comparable to copper with a mass savings of $9.3 \mathrm{~kg}$. The larger diameter (28 AWG) 1350-0 aluminum wire also allows improved manufacturability of a continuous $5 \mathrm{~km}$ length tether. To address concerns on temperature cycling and the effect on the electrical conductivity of the wire, metallic coatings were investigated. The desire was to find a coating that would improve the absorptivity/emissivity $(\alpha / \varepsilon)$ ratio of the bare aluminum, while maintaining the ability of the tether to collect electrons. The coating that was selected is a polymer based coating developed by Triton Systems, Inc. called C-COR, which provides an $\alpha / \varepsilon$ ratio of 1.14 .

The final tether section is $220 \mathrm{~m}$ long and uses a similar seven wire construction as the $5 \mathrm{~km}$ conducting section. Triton Systems, Inc. developed a two-layer insulating coating to prevent any electron reconnection to the tether by the plasma contactor near the Delta stage. The larger outside diameter of this coated wire required the use of a larger diameter braided Kevlar core this section. In addition, this section is also protected by a Kevlar overbraid to prevent any damage to the tether during deployment or throughout the mission. The tether is wound on a core for deployment (Fig. 3).

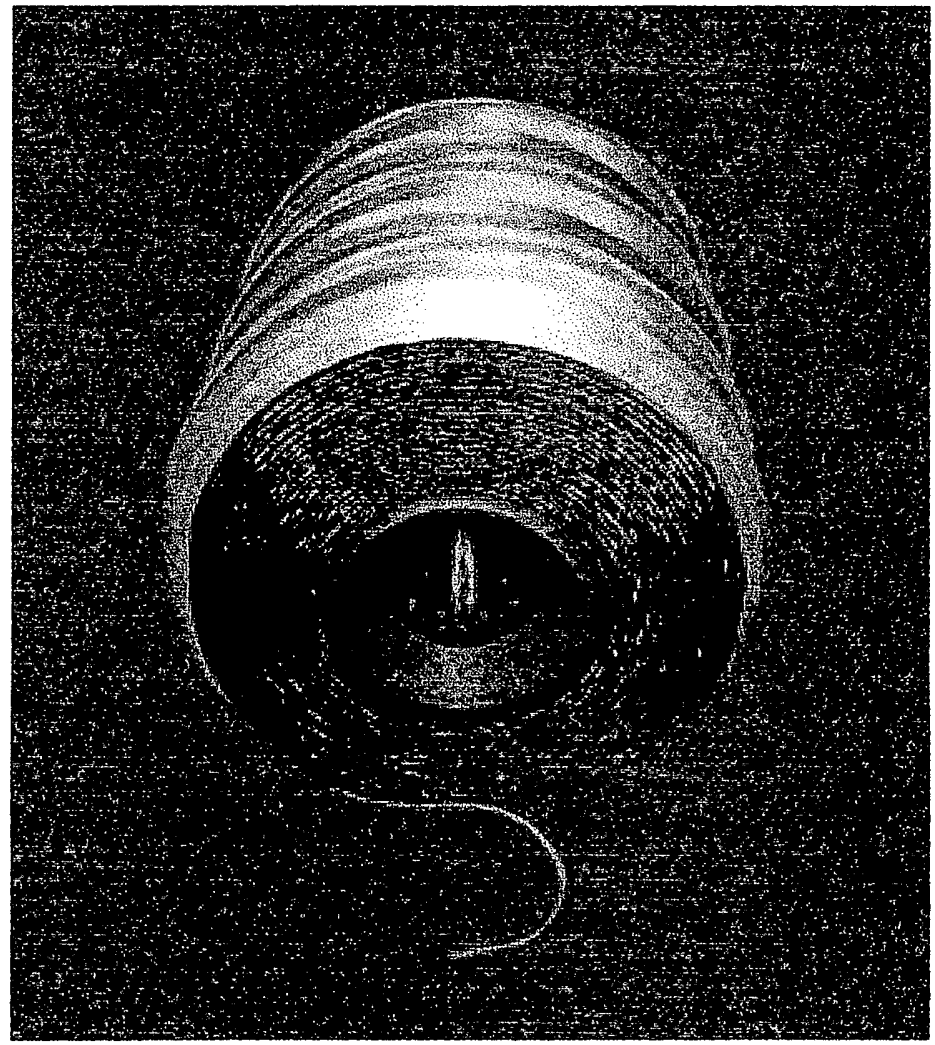

FIGURE 3. ProSEDS Tether Wound on Core.

Consideration of all environments and conditions must be made before developing the requirements for system designs. Some of the conditions may provide conflicting restrictions on the design, so system interactions must be understood and trades conducted early before hardware is designed and built. While there are significant benefits to development time and cost by using existing flight proven hardware, there are aiso substantial constraints imposed 
to the rest of the system in order to make the entire system fit and work together. System design trades constrained by the use of existing designs may limit the use of some design solutions that would provide an overall improved system. When the focus of an experiment is the development and demonstration of a new technology, such as the bare electrodynamic tether it would be beneficial to let the design of the new technology drive the other supporting systems as much as possible.

\section{TETHER MANUFACTURING}

The ProSEDS tether is manufactured in several steps. Production and braiding of the non-conducting section, processing of the wires and coatings for the conducting and insulating section, production and joining of the conducting and insulating tether sections, and splicing of each section together. Due to precise requirements for final dimensions and performance of the ProSEDS tether, the manufacture of the tether requires close monitoring and the use of carefully developed manufacturing procedures. The cooperation of NASA, Tether Applications Inc., and supporting contractor personnel was a key element in developing and producing successful flight tethers for the ProSEDS experiment.

The $10 \mathrm{~km}$ non-conducting tether is a flat braid of Dyneema material using 11 strands of 135 denier fibers braided at 7.5 to 8 picks per inch. The Dyneema material is an ultra high molecular weight $\mathrm{PE}$ which is produced at Western Filament in sections that are spliced together by Tether Applications, Inc. One end of the non-conducting tether is spliced to a much heavier $20 \mathrm{~m}$ length of Kevlar braid used for heat resistance and to prevent the tether from snagging on the endmass to which it is attached. The splice of the Kevlar braid to the Dyneema braid uses several stages of taper to allow a smooth transition from the Kevlar leader to the $10 \mathrm{Km}$ non-conducting tether.

The metallic tether is manufactured in several steps: wire production and coating, Kevlar core fabrication and twisting, wire twisting over Kevlar core, cold welding conducting to insulating wires, overbraiding the insulated tether segment, and application of cross-straps to the conducting section. All of these steps must be completed before the conducting section of tether can be spliced to the non-conducting tether section during winding onto the deployer core. The wire is manufactured and coated by Kanthol in Palm Coast, FL. Kanthol specializes in coating fine magnet wires and wires for surgical processes with the capability to coat aluminum wire down to $0.4 \mathrm{mil}$ in diameter. The of 28 AWG 1350-0 aluminum wire is drawn at Kanthol from 0.125 " wire to 0.0126 " with strict tolerances on wire roundness that is required to perform the coating process. Both the conducting, $\mathrm{CCOR}^{\mathrm{TM}}$ and insulating, $\mathrm{TOR}^{\mathrm{TM}}$ polymer coatings are fabricated by Triton Systems, Inc of Chelmsford, MA. The conductive polymer is a blend of Triton's Colorless Oxygen Resistant (COR) and Polyanaline (Pani) in a blend to provides the necessary conductive, emissive and AO resistant properties for the tether. The insulating coating is polyimide covered with TOR-BP ${ }^{\mathrm{TM}}$ for atomic oxygen protection. The CCOR ${ }^{\mathrm{TM}}$ coating is applied in 12 even increments to the aluminum wire, to produce a final 0.35 mil coating thickness. The insulated coating is applied in two application steps. The polyimide requires two passes through the coating process to build to a 1 mil final thickness with the TOR-BP applied as the final step. The wires are wound onto spools at Kanthol and cut to $6,750 \mathrm{~m}$ per spool for the conductive wires, and $300 \mathrm{~m}$ per spool for the insulated wires.

The wires are shipped to Cortland Cable in Cortland, NY for further processing. Cortland Cable developed and uses the "Hi-Wire" design to manufacture many of their cable products. The Hi-Wire process involves using strands of wire twisted around a polymeric core that is used as the strength member of the cable design. Two different diameter cores of Kevlar TM 49 are used for the ProSEDS tether because the coated wire diameters are different. The smaller diameter conductive section uses a smaller core made by braiding 6 strands of 390 denier material. The larger diameter insulating wires use a core section made by braiding 8 strands of 390 denier material around one strand of 390 denier material. These Kevlar cores are then twisted in a direction opposite the wire twisting direction, so that the wire twisting process removes the initial twist of the Kevlar core allowing the tether to be produced without any induced stresses.

The wire twisting process at Cortand Cable is performed by using seven spools of wire set onto a pay-off rack with magnetic torque control devices used to control the payoff tension. The wires are fed through a twisting die that has 7 equally spaced holes in a circle around a center hole. The Kevlar core is fed through the center hole from a supply spool also with tension control. The wires and core are threaded onto a take-up spool in a Cook twister machine and are pulled and twisted at a rate to apply approximately 3 twists per inch. In order to make a $5 \mathrm{~km}$ section of tether in 
this manner, there must be an understanding of processing requirements and close control of tension and machine operating parameters must be maintained. It is most desirable to produce the entire length of tether in one production run which is a time consuming process that requires watchful care and control. After the conductive section of tether is twisted it must be connected to the insulating section of tether in a manner that provides smooth transition between segments and provides adequate tensile strength for the mission. This is accomplished by performing a splice with the two different sized Kevlar cores to each other and cold welding the two types of wires to each other. The twisting process is stopped for the cold welding procedure that is performed by using a Huestis cold welder to weld each pair of wires together (Fig. 4). The cold welds are staggered in length, by about $3 \mathrm{~cm}$ in order to have a more uniform finish to the tether and a smooth transition between the two different coated wire sections. The cold welded wires have the same or better tensile strength than virgin wire, and they maintain their electrical conductivity. After all seven wires have been cold welded together and the Kevlar core splice is made the twisting process is continued.

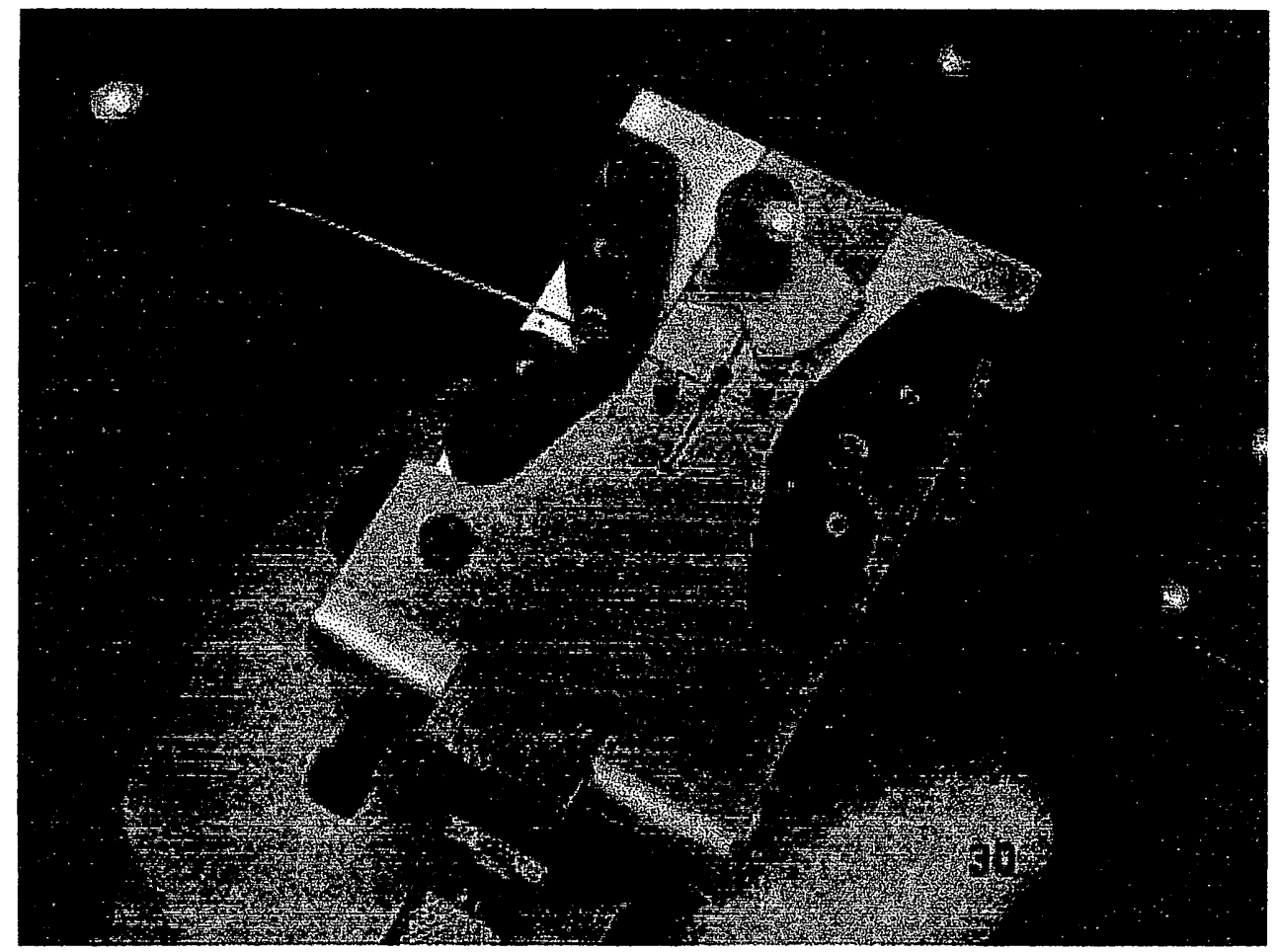

FIGURE 4. Huestis Cold Welder for Splicing Wires on Tether.

The next two steps in tether manufacturing are done to reduce risks to tether damage and to provide improvements to mission success for the ProSEDS experiment. To provide protection from damage or snagging to the insulated section of the tether (which will be closest to the Delta stage after deployment) an overbraid of Kevlar ${ }^{\mathrm{TM}} 49$ is applied. This provides a thin but complete sleeve to cover the insulated tether section. A 16 carrier braider is used to apply this overbraid to the tether. 16 strands of Kevlar ${ }^{\mathrm{TM}} 49380$ denier material are braided with about 13.5 picks per inch. After completion of the overbraiding the tether is packed and shipped to MSFC for cross-strapping and testing. In case one or more of the wires in the $5 \mathrm{~km}$ conductive section of tether is damaged on orbit, a set of 16 cross-straps are applied to the tether to allow the current to continue to flow in the tether. These cross-straps are about $6 \mathrm{~cm}$ long and are made by wrapping a thin copper wire around the tether and then covering the copper wire section with an Aracon fiber overwrap. During the mission if up to 3 wires get broken on the tether the copper provides a means of carrying current from the broken wire side of the cross-strap and distributing it back into all 7 wires on the other side. This will allow the mission to continue even if some damage occurs to the tether. 
After each section of tether is produced they must be spliced together to make a continuous $15 \mathrm{~km}$ long tether. Except for the splices between the insulated and conductive sections, which are done during the wire twisting process at Cortland $\mathrm{Cable}$, all of the tether splices are done during tether winding at Tether Applications. The Kevlar leader to non-conducting Dyneema splice has been discussed previously. The main splice that is performed during winding is the splice between the Dyneema material and the conducting wire tether. During this process the wires are peeled back from the Kevlar core so that the Kevlar core can be spliced to the Dyneema material. Painstaking care must be taken to provide a smooth splice that will withstand the loads during deployment and the mission. After the splice is completed the wires are tucked into the core at staggered distances to provide a smooth transition.

The development of the necessary materials, coatings and manufacturing processes for the ProSEDS tether involved the efforts of many individuals. During the development phase of the project there were many tests conducted on the tether materials, and several production runs made of tether processing. Until a complete tether was manufactured and deployed all of the influences of the manufacturing steps were not clearly understood. Seemingly minor changes to the manufacturing resulting in major changes in the performance and behavior of the finished tether. Adequate time to develop a new technology must be provided for in project scheduling and a clear understanding of the manufacturing processes should be developed before production is initiated.

\section{TETHER TESTING}

It was the philosophy of the ProSEDS Project to conduct extensive testing on all of the hardware including the tethers. An approach of test what you fly, and fly what you test was implemented. Although it was not always possible to test under the exact flight conditions, this approach was used as much as possible. Tests were conducted throughout the development phase of the ProSEDS tether. In addition there were many tests that were incorporated as part of the tether manufacturing process. Finally, deployment tests were performed after the tethers had been made and wound in flight configuration. This section describes some of the significant tether testing that took place.

On individual wire samples, tests were performed in a plasma chamber to verify the CCOR coating's conductive properties. The optical properties of the coatings were measured to verify emissivity. Atomic oxygen tests were conducted on the coatings to verify AO survivability. Finally dielectric breakdown tests were conducted on insulated wire samples to verify allowable voltage standoff capability.

At the completion of the wire manufacturing and coating process and after the twisting process a spark test was performed to detect pinholes or defects in the insulated section. Spark testing is used routinely in the wire industry for this purpose. Spark testing involves high voltage and very low current $(\mu \mathrm{A})$. The spark tester consists of a high voltage detector head and controlling power supply. The spark test for ProSEDS was conducted at $3000 \mathrm{~V}$, which is a factor of two higher than the highest expected EMF during flight. During all of the splicing procedures and the cold welding steps for wire joining, samples were made and tested off-line to insure the breaking strength of the joints. In addition post-joining samples were gathered for further testing. Testing of the cross-straps for current carrying capability and handling ability was conducted prior to final selection of the cross-strap design. High voltage testing of all electric field triple points (such as at the splice between insulated and conductive tether sections) was performed in a vacuum plasma chamber.

After the tethers were wound in the flight configuration, they were vacuum baked to relax the wire residual strain (to reduce the tendency to unspring during low speed deployment) and drive out moisture. They were then vibration tested to demonstrate structural integrity. Finally, deployment tests were conducted in vacuum at various temperatures to ensure that the final tether design would perform as predicted. Tension data was collected and used to verify the performance of the overall tether and winding design. Extensive development deployment tests were conducted as well during the maturation of the tether design. Spark tests of the insulated tether sections were performed after each deployment test. 


\section{CONCLUSIONS}

The ProSEDS tether experiment is scheduled to fly in June of 2002. The new tether design that was developed for this experiment has been designed to perform as needed to collect the electrical current from the space plasma and demonstrate the electrodynamic thrust capability of tethers. In addition several risk mitigation designs were incorporated to improve the probability of mission success. Extensive testing was conducted during the development and production of the tethers for ProSEDS. Much was learned in the process that can be used for future systems that use electrodynamic tether propulsion technology.

\section{ACKNOWLEDGMENTS}

The authors would like to gratefully acknowledge the support and cooperation of personnel at MSFC, Kanthol, Triton Systems, Inc., and Cortland Cable Company. The close collaboration of these organizations was a key element in developing and producing successful flight tethers for the ProSEDS experiment. 